\section{RESIDUAL NECK LUMP}

Sir, I am writing regarding a case seen in the maxillofacial department, where I am currently working as a core trainee. A 68-year-old female patient presented initially to the ENT department with a painful neck 'lump' of a few weeks' duration which had decreased in size with antibiotics prescribed by her GP. A nasendoscopic examination was completed, revealing no abnormalities. Clinical examination revealed a level II lymph node on the right side which was shown by ultrasound examination to be reactive in nature. The patient was reassured and discharged.

Two months later the patient was referred to our department with the same problem. Apart from a slight increase in size, the 'lump' was asymptomatic. Clinical examination revealed a $1.5 \mathrm{~cm}$ hard lump in the right submandibular region, with firmness extending to the lingual aspect of the mandible. Intra-oral examination showed a lone standing, heavily restored wisdom tooth in the lower right quadrant. Further investigation with an orthopantogram (OPG) revealed a periapical radiolucency associated with this tooth and led to a provisional diagnosis of a chronic sinus arising from this periapical infection. An MRI scan confirmed a chronic sinus tract extending from the lingual aspect of the mandible to the neck, almost certainly arising from the chronically infected lower right wisdom tooth. This tooth was removed and the sinus tract excised under local anaesthetic. The residual neck lump resolved soon after the procedure.

This case, and its history, is not uncommon on our clinics. It is of relevance to our ENT colleagues to consider chronic dental infection as a differential diagnosis for neck swelling. Although simple, it can be easily overlooked and lead to an unnecessary delay of treatment for the patient.

J. Harrild, V. Santhanam, by email DOI: 10.1038/sj.bdj.2015.110 compounds. ${ }^{4}$ The local and systemic carcinogenic potential of tattoos remain unclear, being described in literature as purely coincidental. ${ }^{4}$ There are reported cases of malignancy arising from cosmetic tattoos of the lips, but none from the oral mucous membrane. ${ }^{5,6}$ The US Food and Drug Administration has not approved any tattoo pigments for injection into the skin and many pigments used in tattoo inks are industrial-grade colours suitable for printers' ink or automobile paint. ${ }^{2}$ Despite this, faith and fashion seems to be driving popularity. While a counterargument is that tattoos have been practised for a long time, the issue is how they will behave in the oral mucosa and we need to keep a close watch on these cases while also educating our patient about their potential risks.

\section{A. Telang, Penang, Malaysia}

1. Scheinfeld N. Tattoos and religion. Clin Dermatol 2007; 25: 362-365.

2. Skin Artists. Inner lip tattoo - care and health awareness. Available at: http://www.skin-artists. com/inner-lip-tattoo.htm (accessed January 2015).

3. FDA US Food and Drug Administration. Think before you ink: are tattoos safe? Available at: http://www.fda.gov/forconsumers/consumerupdates/ucm048919.htm (accessed January 2015).

4. Kluger N, Koljonen V. Tattoos, inks, and cancer. Lancet Oncol 2012; 13: 161-168.

5. Ortiz A, Yamauchi PS. Rapidly growing squamous cell carcinoma from permanent makeup tattoo. J Am Acad Dermato/ 2009; 60: 1073-1074.

6. Omidian M, Emad-Mostofi N. Basal cell carcinoma arising from traditional tattoo. Arch Iran Med 2009; 12: 198.

DOI: 10.1038/sj.bdj.2015.109

\section{PHARMACEUTICALS}

Tetracyclines and periodontal disease

Sir, I write with regard to the paper Drugs, medications and periodontal disease (BDJ 2014; 217: 411-419) and would like to make some additional points.

Tetracycline antibiotics are one of the oldest classes of broad spectrum antibiotics used. Tetracycline, doxycycline and minocycline are some of the examples of this class of antibiotic. The levels of tetracyclines are found to be higher in gingiva and gingival crevicular fluid than in the serum. In addition to their antibacterial activity, tetracyclines appear to possess anti-inflammatory, anticollagenase, wound-healing properties and also reduce bone loss. Tetracycline inhibits matrix metalloproteinases (MMPs) such as collagenases which have a role in breakdown of connective tissue. Low dose doxycycline also improves periodontal parameters such as clinical attachment levels and probing pocket depths. Doxycycline is more potent than tetracycline in inhibiting MMPs and is found to be safe for administration for three months at $20 \mathrm{mg}$ dose twice a day. Minocycline, in addition to having immunomodulatory properties, stimulates osteoblasts and has a role in enhancing periodontal healing.

Vagish Kumar LS, by email DOI: 10.1038/sj.bdj.2015.111 